Draft Version JANUARY 11, 2021

Preprint typeset using $\mathrm{IAT}_{\mathrm{E} X} \mathrm{X}$ style emulateapj v. 5/2/11

\title{
MASS/RADIUS CONSTRAINTS ON THE QUIESCENT NEUTRON STAR IN M13 USING HYDROGEN AND HELIUM ATMOSPHERES
}

\author{
Catuneanu, A. ${ }^{1,2}$, Heinke, C. O. ${ }^{1}$, Sivakoff, G. R. ${ }^{1}$, Ho, W. C. G. ${ }^{3}$, Servillat, M. ${ }^{4}$ \\ Draft version January 11, 2021
}

\begin{abstract}
The mass and radius of the neutron star (NS) in low-mass X-ray binaries can be obtained by fitting the X-ray spectrum of the NS in quiescence, and the mass and radius constrains the properties of dense matter in NS cores. A critical ingredient for spectral fits is the composition of the NS atmosphere: hydrogen atmospheres are assumed in most prior work, but helium atmospheres are possible if the donor star is a helium white dwarf. Here we perform spectral fits to XMM, Chandra, and ROSAT data of a quiescent NS in the globular cluster M13. This NS has the smallest inferred radius from previous spectral fitting. Assuming an atmosphere composed of hydrogen, we find a significantly larger radius, more consistent with those from other quiescent NSs. With a helium atmosphere (an equally acceptable fit), we find even larger values for the radius.
\end{abstract}

Subject headings: binaries : X-rays — dense matter — stars: neutron — globular clusters: individual (NGC 6205)

\section{INTRODUCTION}

Understanding the interiors of neutron stars (NSs) by measuring their masses and radii is a key goal of highenergy astrophysics (Lattimer \& Prakash 2007). NS masses can be effectively measured for radio pulsars (e.g. Antoniadis et al. 2012) and some X-ray binaries (e.g. Rawls et al. 2011) by radio timing and/or optical/IR radial velocities. However, NS radii are much more complicated to measure. The radius measured at infinity is affected by the NS's gravitational redshift, as $R_{\infty}=$ $R(1+z)=R / \sqrt{1-2 G M /\left(R c^{2}\right)}$ (where $R$ and $M$ refer to the NS values), and in more subtle ways by the surface gravity, magnetic fields, emission anisotropies, and composition. Two key methods of constraining NS radii have involved fitting X-ray burst spectra (Damen et al. 1990), and fitting X-ray spectra of quiescent low-mass X-ray binaries (qLMXBs) containing NSs (Rutledge et al. 1999). Recently, substantial work has been done using RXTE burst spectral measurements to constrain the mass and radius of NSs where the distance can be inferred, though the interpretation of these results has differed due to different choices of assumptions (Boutloukos et al. 2010; Özel et al. 2010; Steiner et al. 2010; Suleimanov et al. 2011; Zamfir et al. 2012; Gallowav \& Lampe 2012).

The qLMXBs typically show thermal blackbody-like radiation and/or a harder nonthermal component, often fit with a power-law of photon index 1 to 2 (Campana et al. 1998). The nonthermal component is of uncertain origin, possibly due to continued accretion or a pulsar wind. The thermal component is better understood, as emission from the NS surface, powered by some combination of continued accretion (Zampieri et al.

\footnotetext{
${ }^{1}$ Dept. of Physics, University of Alberta, Room 238 CEB, Edmonton, AB T6G 2G7, Canada; heinke@ualberta.ca

2 Perimeter Institute, 31 Caroline St., N. Waterloo, ON, N2L 2Y5, Canada

${ }^{3}$ School of Mathematics, University of Southampton, Southampton SO17 1BJ, UK

${ }^{4}$ Laboratoire AIM, CEA Saclay, Bat. 709, 91191 Gif-surYvette, France
}

1995), heat release from the crust deposited by the last outburst (Rutledge et al. 2002b; Degenaar et al. 2011), and heat deposited in the core (created by nuclear fusion in the deep crust during outbursts) that is now leaking out (Brown et al. 1998). Fitting the thermal X-ray spectra of qLMXBs is conceptually simpler than fitting $\mathrm{X}$-ray bursts, as accretion has either stopped or is at a very low level, allowing the stratification of the lightest element to the top of the atmosphere within 30 seconds (Alcock \& Illarionov 1980). Calculations of nonmagnetic hydrogen atmosphere NS spectra generally reach very good agreement with each other and with observations (Rajagopal \& Romani 1996; Zavlin et al. 1996; Heinke et al. 2006; Haakonsen et al. 2012), suggesting that X-ray spectra of qLMXBs can provide useful NS mass/radius constraints.

However, qLMXB radius constraints depend strongly on the distance, and the distances to most qLMXBs are poorly known. One solution is to study qLMXBs in globular clusters, where the distances are typically known to $\sim 5-10 \%$ precision (Brown et al. 1998; Rutledge et al. 2002a). A number of qLMXBs have been studied in globular clusters (see Guillot et al. 2009, for a review), but only a few have sufficiently high-quality spectra to provide interesting constraints on the equation of state; these include X5 and X7 in 47 Tuc (Heinke et al. 2003a, 2006), and the qLMXBs in $\omega$ Cen (Rutledge et al. 2002a; Gendre et al. 2003b; Webb \& Barret 2007), NGC 6397 (Grindlav et al. 2001; Guillot et al. 2011a), M28 (Becker et al. 2003; Servillat et al. 2012), M13 (Gendre et al. 2003a; Webb \& Barret 2007), and NGC 6553 (Guillot et al. 2011b). Interestingly, two of these constraints (X7 in 47 Tuc, vs. M13) disagree with each other at the $99 \%$ confidence level, motivating efforts to understand the discrepancy. Uncertainties in the pileup correction used for 47 Tuc X7 (Davis 2001) might drive the discrepancy, suggesting a deep observation of 47 Tuc with a smaller frame time to eliminate pileup. Alternatively, the discrepancy may be due to differing atmospheres. 
A large fraction of bright persistent or transient LMXBs in globular clusters have orbital periods less than one hour (so-called "ultracompact" systems, 5 of the 10 systems with known periods), requiring degenerate white dwarf companions; such systems can be easily created through dynamical interactions in globular clusters (Deutsch et al. 2000; Ivanova et al. 2005). Ultracompact systems are likely to accrete material without hydrogen, since the donor star is (for the most typical evolutionary tracks) devoid of hydrogen, though spallation of infalling material to create hydrogen is possible (Bildsten et al. 1992). Several characteristics of X-ray bursts differ between ultracompact vs. long-period LMXBs; the ratio of integrated persistent to burst fluence (much larger for ultracompact systems, indicating a lack of $\mathrm{H}$ ), the existence of intermediate-long bursts at low mass transfer rates in ultracompacts (ignition of large He columns without $\mathrm{H}$ bursts), higher Eddington limits from ultracompacts than long-period sources (where distances are independently estimated from their globular cluster locations), and consistently short bursts at moderate mass transfer rates in ultracompacts (when mixed $\mathrm{H} / \mathrm{He}$ ignition is expected if $\mathrm{H}$ is present) (Cumming 2003; in't Zand et al. 2005; Gallowav et al. 2008, 2010). Thus it seems likely that many globular cluster qLMXBs may have atmospheres composed of $\mathrm{He}$, or $\mathrm{C}$, depending on the nature of the donor star.

The possibility of differing atmospheric composition for globular cluster NS qLMXBs may explain the discrepancy between globular cluster qLMXB mass/radius constraints, since fits with He atmosphere models give larger radii than $\mathrm{H}$ atmosphere fits (Ho \& Heinke 2009). We used the qLMXB in M28 as a first example of the differences in mass/radius constraints from the two models (Servillat et al. 2012). Here we consider the qLMXB with the smallest known radius constraint, the M13 qLMXB. M13 has been studied by ROSAT's PSPC and HRI cameras, which detected an X-ray source (labeled Ga) in the core of the cluster (Fox et al. 1996; Verbunt 2001), the target of this study. Gendre et al. (2003a) used XMM to identify another source, 15" to the NW of $\mathrm{Ga}$, which may contribute to the ROSAT PSPC and XMM spectra of Ga, and showed that Ga's spectrum was consistent with a hydrogen-atmosphere NS. Webb \& Barret (2007) then calculated detailed constraints on its mass and radius, along with XMM studies of two other NSs in globular clusters. Servillat et al. (2011) used ground-based optical (Faulkes Telescope North), Chandra X-ray, and Hubble Space Telescope data to identify the source NW of Ga (their star 4, or X6; we use the latter name) as a cataclysmic variable (CV) experiencing a dwarf nova outburst. Some additional results from the archival Chandra observations of M13 (PI: Lewin) have been published (Poolev \& Hut 2006; Hui et al. 2009), but spectral analysis of Chandra data on the NS qLMXB has not yet been published.

\section{OBSERVATIONS AND DATA REDUCTION}

Our principal dataset is a pair of XMM-Newton observations obtained in Jan. 2002 using the EPIC cameras using the medium filters. We also use a pair of Chandra observations taken in March 2006 using the ACIS-S detector in FAINT mode, and a ROSAT observation taken in 1992 in pointing mode (see Table 1).
Table 1 Observations

\begin{tabular}{ccccccc}
\hline \hline Mission & ObsID & Date & Instr. & $\begin{array}{c}\text { GTI } \\
(\mathrm{s})\end{array}$ & $\begin{array}{c}\text { GTI2 } \\
(\mathrm{s})\end{array}$ & Counts \\
\hline CXO & 7290 & $2006-03-09$ & ACIS-S & 27894 & - & 300 \\
CXO & 5436 & $2006-03-11$ & ACIS-S & 26799 & - & 305 \\
XMM & 85280301 & $2002-01-28$ & MOS1 & 18044 & 14814 & 86 \\
& & & MOS2 & 18051 & 15070 & 76 \\
& & & PN & 14353 & 10338 & 283 \\
XMM & \multirow{2}{*}{85280801} & \multirow{2}{*}{$002-01-30$} & MOS1 & 16340 & 13537 & 126 \\
& & & MOS2 & 16630 & 14004 & 51 \\
ROSAT & \multirow{2}{*}{300181} & \multirow{2}{*}{$1992-09$} & PSPCB & 45872 & - & 426 \\
\end{tabular}

Note. - Observations of M13 used in this analysis, with GTI exposure times for XMM data quoted both with (GTI2) and without (GTI) aggressive background flare removal. The number of counts in the extraction regions are given for our extractions without aggressive flare removal

The Chandra data were reduced using Chandra Interactive Analysis of Observations (CIAO) v.4.45 and Chandra Calibration Database (CALDB) v4.4.8. We reprocessed the data with the chandra_repro reprocessing script to apply the latest calibration updates and bad pixel files, and filtered the data to the energy range $0.3-10.0 \mathrm{keV}$. The Chandra data showed no strong background flaring, so we included all data.

The position of the qLMXB in M13 (using wavdetect on the combined Chandra data) is R.A. $=16^{h} 41^{m} 43.77^{s}$, decl. $=+36^{\circ} 27^{\prime} 57.64^{\prime \prime}$, consistent with the position given in Servillat et al. (2011) for their Chandra source X7. The neighboring source, at R.A. $=16^{h} 41^{m} 42.47^{s}$, decl. $=$ $+36^{\circ} 28^{\prime} 07.29^{\prime \prime}$, is source X6 from Servillat et al. (2011) (see Fig. 1), who showed it to be a CV exhibiting a dwarf nova eruption. Its X-ray luminosity is $L_{X}(0.5$ $10)=2.2_{-1.3}^{+1.3} \times 10^{32} \mathrm{ergs} / \mathrm{s}$, for a spectral shape consistent with an absorbed power-law of photon index $\Gamma=1.4 \pm 0.4$ (from simple spectral fits to the Chandra spectra). The spectra of the qLMXB were extracted using circles with radii of $\sim 2^{\prime \prime}$. The specextract script generated the corresponding auxiliary response files (ARFs) and redistribution matrix files (RMFs).

The XMM-Newton data were reduced with the Science Analysis System (SAS) v11.0.0. We repipelined the MOS data using emchain and the PN data with epchain before applying the relevant filters (e.g. patterns $0-12$ for MOS, $0-4$ for $\mathrm{pn}$ ). We also repipelined the MOS and PN data using the 2007 calibration data, as available to Webb \& Barret (2007), for comparison to their analysis (see §3.2). Both XMM observations revealed signs of background flaring, affecting roughly $1 / 3$ of the observations. Since the background flares are not extremely bright, we judge that spectra including all data attain a higher signal-to-noise ratio than if the flares were filtered out. We do, however, filter out flares to match the GTIs of Webb \& Barret (2007) when reproducing their analysis. In this case, we use filters of 5 and 10 counts per second for the two MOS datasets, and 30 and 50 counts per second for the pn data.

Circular regions with approximate radii of $9.5^{\prime \prime}$ (to exclude photons from the CV X6) were used to extract the spectra of the NS qLMXB from the XMM observa-

\footnotetext{
5 http://cxc.cfa.harvard.edu/ciao/
} 


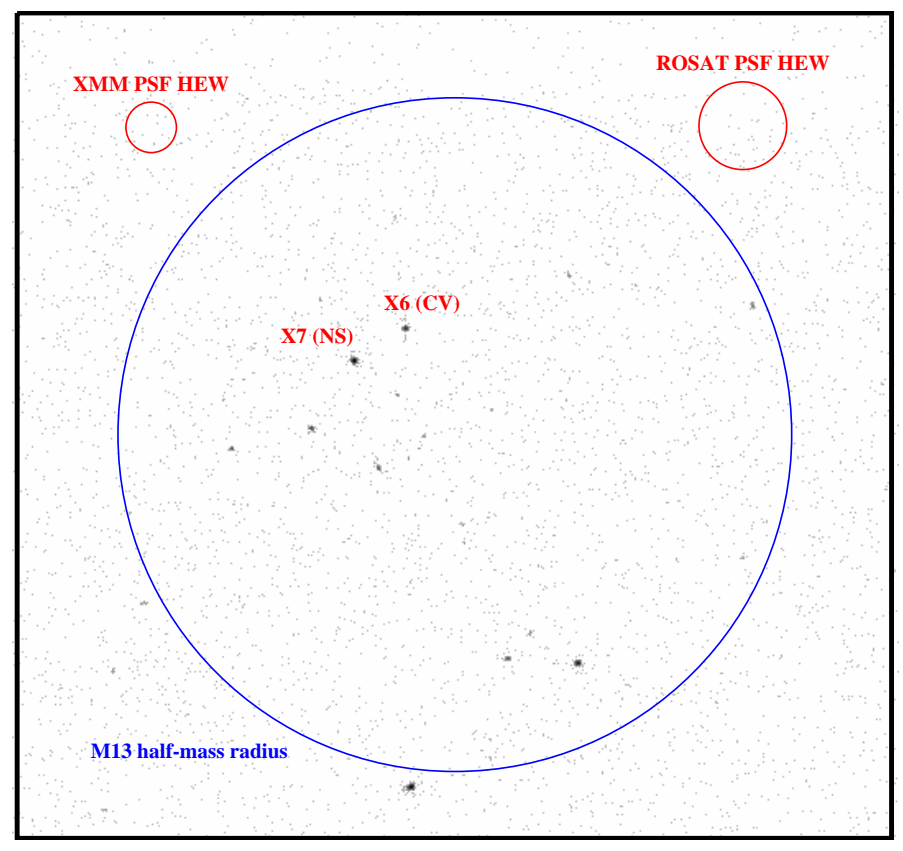

Figure 1. Combined Chandra 0.5-6 keV image of M13, showing the two relevant sources X7 (the NS qLMXB) and X6 (the nearby CV). The radius enclosing half of M13's mass (1.69') is indicated, as are circles roughly indicating the half-equivalent-width (HEW, enclosing $50 \%$ of the energy) of the point-spread functions of the XMM-Newton pn (radius 7.6") and ROSAT PSPC (radius 0.22') detectors.

tions, except for the analysis in $\S 3.2$, where we used a larger $\left(24^{\prime \prime}\right)$ extraction circle, with a $13^{\prime \prime}$ circle around $\mathrm{X} 6$ excluded, to match the extraction region used in Webb \& Barret (2007). ARFs and RMFs were generated with the SAS tasks arfgen and rmfgen. We combined the two MOS spectra in each XMM observation using the addspec tool to achieve better statistics, except for the $\S 3.2$ analysis. Finally, all spectra were grouped to at least 20 counts per bin.

We used the XSELECT tool in HEASOFT6 (v6.12) to reduce the ROSAT data. 7. The NS spectrum was extracted from a circular region of approximately $47^{\prime \prime}$ in radius and then grouped to twenty counts per bin with no filters applied, using the on-axis response matrices.

\section{DATA ANALYSIS AND RESULTS}

We first fit the hydrogen NS atmosphere to the combined XMM, Chandra and ROSAT dataset, and calculate the constraints on the NS mass and radius. We then attempt to reproduce the Webb \& Barret (2007) result, using the data and calibrations available then. Finally, we fit helium atmosphere models, and report the NS constraints in that case. In all cases, we assume a distance to M13 of $7.7 \mathrm{kpc}$ Harris (1996) (2003 revision), and a minimum $N_{H}$ of $1.1 \times 10^{20} \mathrm{~cm}^{-2}$ (Harris 1996).

\subsection{NSATMOS Applied to Chandra, XMM and ROSAT Data}

We simultaneously fit the XMM-Newton, Chandra, and ROSAT spectra to an absorbed hydrogen atmosphere NS model, NSATMOS (

\footnotetext{
6 http://heasarc.gsfc.nasa.gov/docs/software/lheasoft/
} ${ }^{7}$ http://heasarc.gsfc.nasa.gov/docs/rosat/ros_xselect_guide/xselect_ftoplasstal $\left.\sim 1.4 M_{\odot}\right)$. We attempted to replicate their fit by
NS mass was fixed to $1.4 M_{\odot}$ for our initial fitting (relaxed below). We use a normalization constant to allow for the relative differences between each detector. The cross-calibration work of Tsuiimoto et al. (2011) indicates that the MOS detectors give normalizations matching the average of several observatories, so we fix the MOS normalization to 1.0 and allow the pn normalization to float. Fixing the pn normalization to 1.0 instead leads to slightly $(\sim 5 \%)$ larger inferred NS radii, while fixing the Chandra normalization leads to slightly smaller inferred radii.

We also included a bremsstrahlung model when fitting the ROSAT data, as ROSAT's PSPC camera was unable to resolve the NS and X6 as individual point sources (see Fig. 1), following Webb \& Barret (2007). In the XMM and Chandra data sets, these sources were resolved, with no evidence for a contribution by X6 to the NS spectrum (again agreeing with Webb \& Barret 2007). Since X6 provided few counts in the ROSAT data, and ROSAT has relatively poor spectral resolution (and no information above $2.5 \mathrm{keV}$ ), we fixed $k T$ for the bremsstrahlung model of X6 to $4.5 \mathrm{keV}$ in our fits, consistent with Webb \& Barret (2007) and with simple fits to its Chandra spectra. Omitting this component had relatively small effects, but our ROSAT fits include this component as it should be present; its fitted $0.5-10 \mathrm{keV}$ flux was $2 \times 10^{-13} \mathrm{ergs} / \mathrm{cm}^{2} / \mathrm{s}$.

We also tried adding a power-law component to the model describing all data, as found for many qLMXBs, with a photon index fixed at either 1.5 or 2 (Campana et al. 1998). Such a power-law did not significantly improve the fit, and the normalization was consistent with zero within $90 \%$ confidence. To get the clearest constraints on a power-law component, we fit the 0.3-8 unbinned Chandra spectra with the C-statistic in XSPEC. This finds the power-law normalization to be consistent with zero, with its upper limit to be $8 \%$ of the total $0.5-10 \mathrm{keV}$ flux, consistent with power-law upper limits from other globular cluster NS qLMXBs (Heinke et al. 2003b). We omit the power-law component from our fitting below.

We have fit the model to both flare-filtered and unfiltered XMM data. While the flare-filtered data have less background contamination, they contain only $2 / 3$ of the source photons, so the $\mathrm{S} / \mathrm{N}$ ratio is similar. Table 2 compiles results of fits to both cases, with the NS mass held fixed at $1.4 M_{\odot}$. We found no evidence for additional absorption in the binary. We show a spectral fit to Chandra, ROSAT and unfiltered XMM data in Fig. 2.

We then allow the mass of the NS to vary, finding a best fit with NS mass $1.5 M_{\odot}$ and radius $10.2 \mathrm{~km}$ (using the full XMM dataset). We show a contour plot of $\chi^{2}$, generated with the steppar command, over mass and radius in Fig. 3. The filtered XMM dataset gave similar contours, though the best-fit NS mass slid down the minimal $\chi^{2}$ valley to reach the lower bound of the NSATMOS model at $0.5 M_{\odot}$.

\subsection{NSATMOS Applied to XMM and ROSAT Data}

We noticed that our hydrogen-atmosphere model fit, above, did not reach similar results on the size of the M13 NS to the work of Webb \& Barret (2007), who require a relatively small NS $(<11 \mathrm{~km}$ at $90 \%$ confidence for 
Table 2

H Atm. Fits, CXO, XMM \& ROSAT

\begin{tabular}{|c|c|}
\hline Parameter & Value \\
\hline \multicolumn{2}{|c|}{ XMM Filtered for Flares } \\
\hline $\begin{array}{l}\text { Intrinsic } N_{H} \\
\text { NSATMOS } \log _{10} T \\
\text { NSATMOS } R \\
\text { Reduced } \chi^{2} / \text { dof } \\
\text { Null Hyp. Prob. } \\
\end{array}$ & $\begin{array}{c}0.0_{-0.0}^{+0.8} \times 10^{20} \mathrm{~cm}^{-2} \\
5.99_{-0.05}^{+0.05} \\
11.7_{-2.2}^{+1.9} \mathrm{~km} \\
0.7551 / 73 \\
0.94 \\
\end{array}$ \\
\hline \multicolumn{2}{|c|}{ Without Flare Filtering } \\
\hline $\begin{array}{l}\text { Intrinsic } N_{H} \\
\text { NSATMOS } \log _{10} T \\
\text { NSATMOS } R \\
\text { Reduced } \chi^{2} / \text { dof } \\
\text { Null Prob. }\end{array}$ & $\begin{array}{c}0.0_{-0.0}^{+0.7} \times 10^{20} \mathrm{~cm}^{-2} \\
6.00_{-0.04}^{+0.06} \\
10.6_{-2.2}^{+2.1} \mathrm{~km} \\
0.8337 / 84 \\
0.86\end{array}$ \\
\hline
\end{tabular}

Note. - Chandra, ROSAT and XMM-Newton (flarefiltered and unfiltered) data fit to an NSATMOS hydrogenatmosphere NS model. A bremsstrahlung model was added to the ROSAT spectrum to model X6's spectrum, included in the ROSAT extraction. The second fit corresponds to Fig. 3 for a NS mass fixed to $1.4 M_{\odot}$. The $N_{H}$ quoted is any intrinsic $N_{H}$ in the binary, in addition to the (fixed) cluster value of $1.1 \times 10^{20} \mathrm{~cm}^{-2}$.

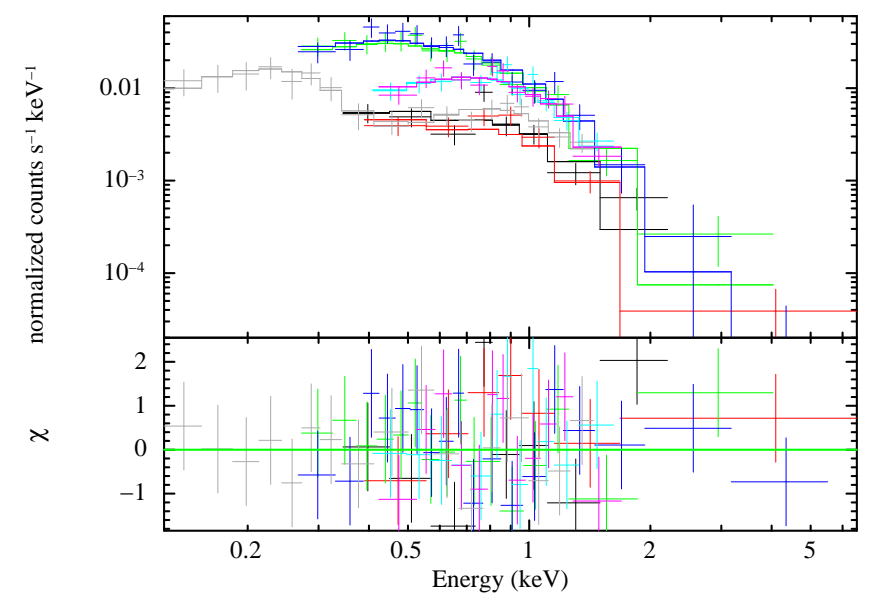

Figure 2. Spectra of M13 qLMXB, including unfiltered XMM MOS (red and black) and pn (blue and green), Chandra (light blue and magenta), and ROSAT (light gray) data, fit with the NSATMOS hydrogen-atmosphere model. (The ROSAT model also contains a bremsstrahlung component, for the unresolved CV X6.) The lower portion of the diagram depicts $\chi^{2}$ residuals.

reprocessing and extracting the XMM data as in Webb et al. (see $\S 2$ above). For a NS mass fixed to $1.4 M_{\odot}$, we obtain the fit in Table 3.

When allowing the mass to vary, we find a best-fit NS mass of $1.3 M_{\odot}$ and a $10.0 \mathrm{~km}$ radius, not dissimilar to the best fit of Webb et al. (their Table 2). However, our contours (Fig. 4) are rather less constraining than those in Webb et al.'s Fig. 6. Webb et al.'s quoted best fits lie very close to their upper $90 \%$ confidence contour lines. We do not see such behavior in any well-behaved chi-squared contour plots of the M13 qLMXB, or any other NSs in our experience. The uncertainties on radius quoted in their Table 2 also seem unusually narrow.

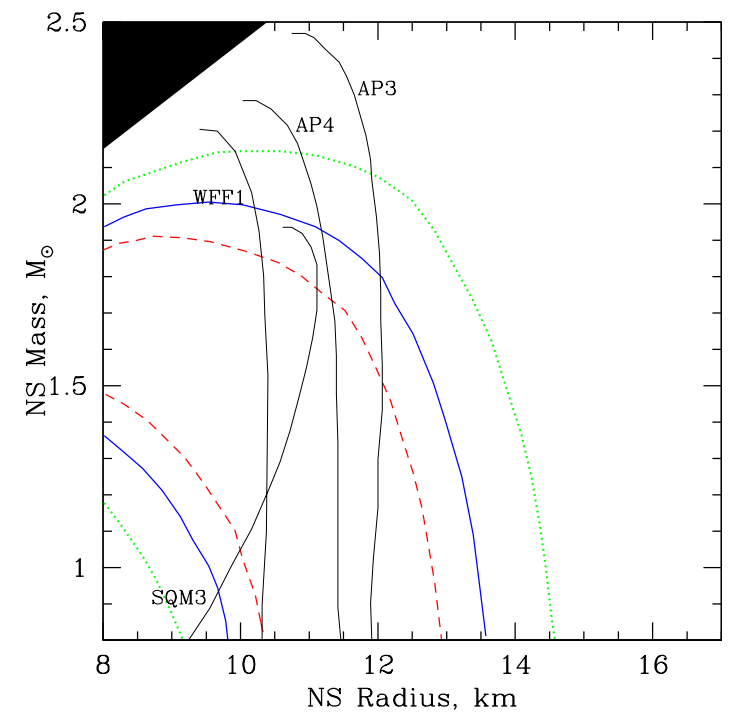

Figure 3. Mass vs radius confidence contours for the non-flarefiltered fit to XMM, Chandra and ROSAT data with the NSATMOS hydrogen atmosphere. The solid (blue), dotted (green), and dashed (red) lines represent 90\%, 99\%, and $68 \%$ confidence contours respectively. The upper left section (with $R<2.8 M$, Lindblom 1984) is shaded to indicate its inaccessibility for any NS, as any equation of state would require the sound speed to exceed c.

Table 3

H Atm. Fits, XMM \& ROSAT

\begin{tabular}{lc}
\hline \hline \multicolumn{1}{c}{ Parameter } & Value \\
\hline Intrinsic $N_{H}$ & $0.0_{-0.0}^{+0.6} \times 10^{20} \mathrm{~cm}^{-2}$ \\
NSATMOS $\log _{10} T$ & $6.04_{-0.07}^{+0.06}$ \\
NSATMOS $R$ & $9.5_{-1.5 *}^{+3.0} \mathrm{~km}$ \\
Reduced $\chi^{2} /$ dof & $0.8595 / 68$ \\
Null Hyp. Prob. & 0.79 \\
& \\
\hline
\end{tabular}

Note. - ROSAT and flare-filtered XMM spectra of the M13 NS fit by an NSATMOS model, with a bremsstrahlung component included to fit X6 in the ROSAT data. This corresponds to Fig. 4 for a NS mass fixed to $1.4 M_{\odot}$. Errors marked with * indicate the parameter hits a hard limit of the model. The $N_{H}$ quoted is any intrinsic $N_{H}$ in the binary, in addition to the (fixed) cluster value of $1.1 \times 10^{20} \mathrm{~cm}^{-2}$.

\subsection{A Helium Atmosphere}

Finally, we consider the effects of a helium atmosphere on the inferred NS properties. We used the helium atmosphere model described in Ho \& Heinke (2009) to fit the data as described in $\S 3.1$. We present fits with the NS mass fixed to $1.4 M_{\odot}$, using flare-filtered vs. unfiltered XMM data, in Table 4, and plot mass vs. radius contours in Fig. 5.

Although the flare-filtered data have a slightly better fit to the hydrogen model $\left(\delta \chi^{2}=1.5\right.$ for the same degrees of freedom), the helium model fit is still acceptable. The fits to the unfiltered XMM data produce essentially indistinguishable $\chi^{2}$ values for helium or hydrogen fits. Thus, we cannot determine from the current data whether a hydrogen or helium atmosphere is the correct one, and thus which mass-radius contours are appropriate for the 


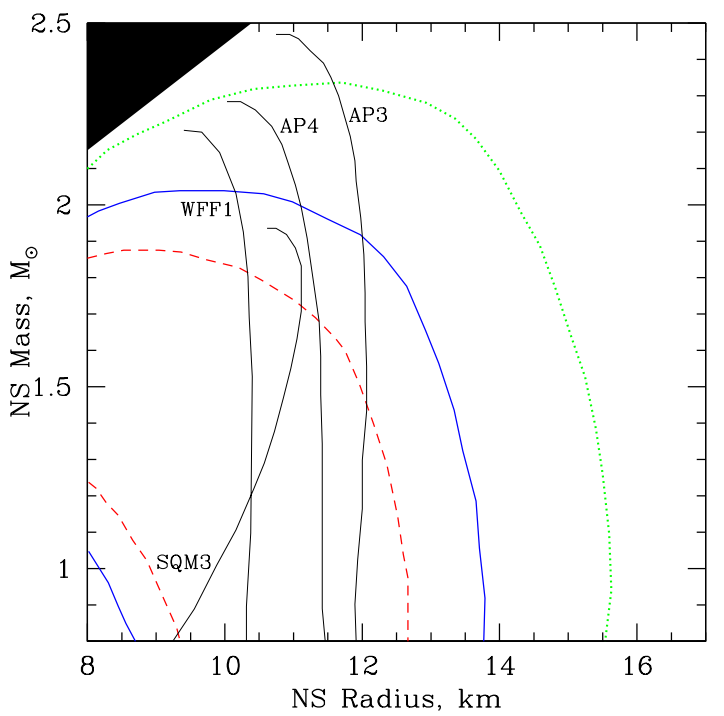

Figure 4. Mass vs. radius contours from the NSATMOS hydrogen-atmosphere fit to XMM and ROSAT data processed following Webb \& Barret (2007). The solid (blue), dotted (green) and dashed (red) lines represent 90\%, 99\%, and 68\% confidence contours respectively.

Table 4

Helium Atm. Fits, CXO, XMM \& ROSAT

\begin{tabular}{|c|c|}
\hline Parameter & Value \\
\hline \multicolumn{2}{|c|}{ XMM Filtered for Flares } \\
\hline $\begin{array}{l}\text { Intrinsic } N_{H} \\
\text { He NS } \log _{10} T \\
\text { He NS } R \\
\text { Reduced } \chi^{2} / \text { dof } \\
\text { Null Prob. } \\
\end{array}$ & $\begin{array}{c}0.4_{-0.4}^{+0.9} \times 10^{20} \mathrm{~cm}^{-2} \\
5.94_{-0.04}^{+0.05} \\
14.6_{-3.1}^{+3.5} \mathrm{~km} \\
0.7753 / 73 \\
0.92 \\
\end{array}$ \\
\hline \multicolumn{2}{|c|}{ Without Flare Filtering } \\
\hline $\begin{array}{l}\text { Intrinsic } N_{H} \\
\text { He NS } \log _{10} T \\
\text { He NS } R \\
\text { Reduced } \chi^{2} / \text { dof } \\
\text { Null Prob. }\end{array}$ & $\begin{array}{c}0.2_{-0.2}^{+0.8} \times 10^{20} \mathrm{~cm}^{-2} \\
5.96_{-0.05}^{+0.05} \\
12.8_{-1.2}^{+3.2} \mathrm{~km} \\
0.8341 / 84 \\
0.86\end{array}$ \\
\hline
\end{tabular}

Note. - Fits to our helium atmosphere model. The second fit corresponds to Fig. 5 for a NS mass fixed to $1.4 M_{\odot}$ (see text). The $N_{H}$ quoted is the intrinsic $N_{H}$ in the binary, added to the (fixed) cluster value of $1.1 \times 10^{20} \mathrm{~cm}^{-2}$.

\section{M13 qLMXB.}

\section{DISCUSSION}

Whether a NS qLMXB hosts a hydrogen or helium atmosphere affects the mass and radius constraints inferred from fitting its quiescent X-ray spectrum. For the M13 qLMXB, for instance, the best-fit NS radius increases by $\sim 2 \mathrm{~km}$ with a helium atmosphere. Although ultracompact LMXBs are known to be common in globular clusters, it is not certain whether ultracompact qLMXBs will display pure helium (or, for hybrid white dwarf donors, carbon; see Nelemans \& Jonker 2010) atmospheres, which introduces uncertainty into calcula-

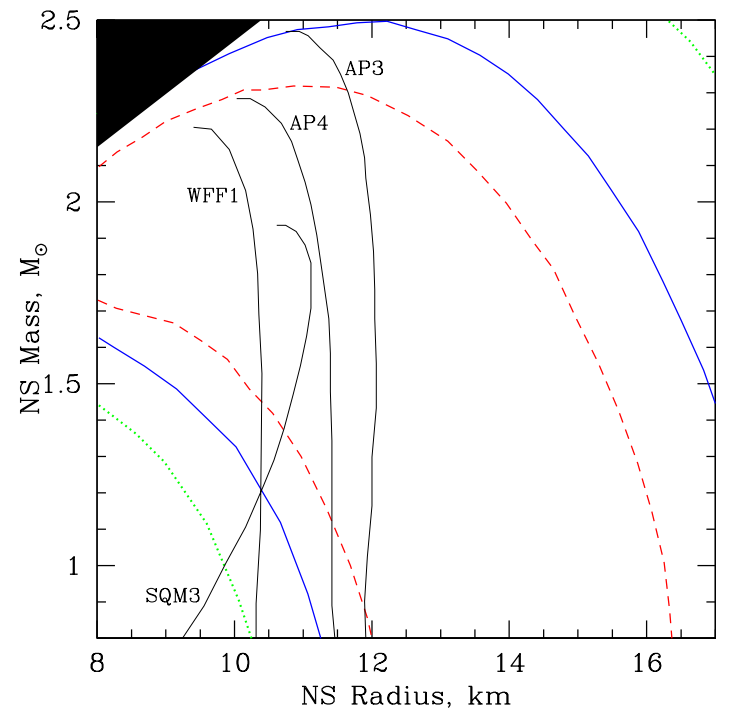

Figure 5. Mass vs. radius contours when fitting the Chandra, non-flare-filtered XMM, and ROSAT data with a helium atmosphere model.

tions of the radii of any NSs that may be in ultracompact qLMXBs.

There are several ways to distinguish ultracompact qLMXBs from normal qLMXBs. Detection of hydrogen in the spectrum, or (narrow-band) photometry, of the companion can provide a clear distinction; this proves that the qLMXB in $\omega$ Cen has a hydrogen atmosphere (e.g. Haggard et al. 2004). Measurement of the orbital period (e.g. through eclipses or pulsation timing in outburst) allows clear discrimination, as done for the eclipsers W37 and X5 in 47 Tuc (Heinke et al. 2005, 2003a) and the millisecond pulsars SAX J1748.9-2021 and NGC 6440 X-2 in NGC 6440 (Altamirano et al.2008, 2010). The energetics and duration of X-ray bursts, when these can be clearly attributed to a particular qLMXB, may provide evidence for or against the presence of hydrogen; e.g., the surface of the Terzan 5 transient EXO $1745-248$ is known to contain hydrogen Galloway et al. 2008). For the M13 qLMXB, narrow-band photometry should be the first project. Finding evidence of $\mathrm{H}-\alpha$ emission from an optical counterpart, using the Chandra position and archival (or new, deeper) Hubble Space Telescope observations of M13, would prove that the NS photosphere is made of hydrogen.

Another concern is the presence of cross-calibration differences between the various detectors used for this type of work; Chandra's ACIS detector, and XMM's pn and MOS detectors. Tsujimoto et al. (2011) found that the XMM pn detector gives 1-8 keV fluxes $6.5 \%$ lower than the MOS detectors in simultaneous observations, while Chandra's ACIS-S3 detector averages $11.6 \%$ higher fluxes. Our analysis gave average XMM pn normalizations $5.5 \%$ higher, and average Chandra ACIS-S3 normalizations $12.5 \%$ higher, than the MOS normalizations, consistent with other Chandra/XMM cross-calibration in the $0.5-2 \mathrm{keV}$ range (Nevalainen et al. 2010). Fixing the other instruments, instead, to have normalization equal to one would systematically increase the inferred NS radius by roughly 3 and $6 \%$, respectively. It is not obvious which detector's calibration is more accurate, which sug- 
gests a $\sim 5 \%$ systematic uncertainty in all such NS radius measurements.

Our result affects inferences of the equation of state of NSs, as the Webb \& Barret (2007) analysis of the M13 NS gave some of the tightest constraints, consistent with the Ozel et al. (2010) meta-analysis of X-ray burst results that preferred a relatively small NS radius under $10 \mathrm{~km}$. Our re-analysis does not support small radii for a hydrogen atmosphere fit. For a helium atmosphere, the radius would be even larger, $>10 \mathrm{~km}$ at 1 sigma for typical NS masses. Our results are consistent with the Steiner et al. (2010) meta-analysis of NS bursters and qLMXBs, which preferred a NS radius between $11-12 \mathrm{~km}$. Steiner et al. 2012 explicitly show that their results are robust against the removal of the Webb \& Barret (2007) M13 qLMXB constraints. Additional high-quality NS constraints would be very valuable in further constraining the NS equation of state, but should consider uncertainties in atmospheric composition and absolute flux calibration.

We thank N. Webb and S. Morsink for discussions. $\mathrm{COH}$ and GRS are supported by NSERC Discovery Grants, and $\mathrm{COH}$ also by an Ingenuity New Faculty Award. WCGH appreciates the use of the computer facilities at KIPAC. WCGH acknowledges support from STFC in the UK.

\section{REFERENCES}

Alcock, C. \& Illarionov, A. 1980, 235, 534

Altamirano, D., Casella, P., Patruno, A., Wijnands, R., \& van der Klis, M. 2008, ApJ, 674, L45

Altamirano, D. et al. 2010, ApJ, 712, L58

Antoniadis, J. et al. 2012, MNRAS, 423, 3316

Becker, W. et al. 2003, ApJ, 594, 798

Bildsten, L., Salpeter, E. E., \& Wasserman, I. 1992, ApJ, 384, 143

Boutloukos, S., Miller, M. C., \& Lamb, F. K. 2010, ApJ, 720, L15

Brown, E. F., Bildsten, L., \& Rutledge, R. E. 1998, ApJ, 504, L95

Campana, S., Colpi, M., Mereghetti, S., Stella, L., \& Tavani, M. 1998, A\&A Rev., 8, 279

Cumming, A. 2003, ApJ, 595, 1077

Damen, E., Magnier, E., Lewin, W. H. G., Tan, J., Penninx, W., \& van Paradijs, J. 1990, A\&A, 237, 103

Davis, J. E. 2001, ApJ, 562, 575

Degenaar, N. et al. 2011, MNRAS, 412, 1409

Deutsch, E. W., Margon, B., \& Anderson, S. F. 2000, ApJ, 530, L21

Fox, D., Lewin, W., Margon, B., van Paradijs, J., \& Verbunt, F. 1996, MNRAS, 282, 1027

Galloway, D. K. \& Lampe, N. 2012, ApJ, 747, 75

Galloway, D. K., Muno, M. P., Hartman, J. M., Psaltis, D., \& Chakrabarty, D. 2008, ApJ Supp, 179, 360

Galloway, D. K., Yao, Y., Marshall, H., Misanovic, Z., \& Weinberg, N. 2010, ApJ, 724, 417
Gendre, B., Barret, D., \& Webb, N. 2003a, A\&A, 403, L11

Gendre, B., Barret, D., \& Webb, N. A. 2003b, A\&A, 400, 521

Grindlay, J. E., Heinke, C. O., Edmonds, P. D., Murray, S. S., \& Cool, A. M. 2001, ApJ, 563, L53

Guillot, S., Rutledge, R. E., Bildsten, L., Brown, E. F., Pavlov, G. G., \& Zavlin, V. E. 2009, MNRAS, 392, 665

Guillot, S., Rutledge, R. E., \& Brown, E. F. 2011a, ApJ, 732, 88

Guillot, S., Rutledge, R. E., Brown, E. F., Pavlov, G. G., \& Zavlin, V. E. 2011b, ApJ, 738, 129

Haakonsen, C. B., Turner, M. L., Tacik, N. A., \& Rutledge, R. E. 2012, ApJ, 749, 52

Haggard, D. et al. 2004, ApJ, 613, 512

Harris, W. E. 1996, AJ, 112, 1487

Heinke, C. O., Grindlay, J. E., \& Edmonds, P. D. 2005, ApJ, 622,556

Heinke, C. O., Grindlay, J. E., Lloyd, D. A., \& Edmonds, P. D. 2003, ApJ, 588, 452

Heinke, C. O., Grindlay, J. E., Lugger, P. M., Cohn, H. N., Edmonds, P. D., Lloyd, D. A., \& Cool, A. M. 2003, ApJ, 598, 501

Heinke, C. O., Rybicki, G. B., Narayan, R., \& Grindlay, J. E. 2006, ApJ, 644, 1090

Ho, W. C. G. \& Heinke, C. O. 2009, Nature, 462, 71

Hui, C. Y., Cheng, K. S., \& Taam, R. E. 2009, ApJ, 700, 1233

in't Zand, J. J. M., Cumming, A., van der Sluys, M. V., Verbunt, F., \& Pols, O. R. 2005, A\&A, 441, 675

Ivanova, N., Rasio, F. A., Lombardi, Jr., J. C., Dooley, K. L., \& Proulx, Z. F. 2005, ApJ, 621, L109

Lattimer, J. M. \& Prakash, M. 2007, Phys. Rep., 442, 109

Lindblom, L. 1984, ApJ, 278, 364

Nelemans, G. \& Jonker, P. G. 2010, New Astron. Rev., 54, 87

Nevalainen, J., David, L., \& Guainazzi, M. 2010, A\&A, 523, A22

Özel, F., Baym, G., \& Güver, T. 2010, Phys. Rev. D, 82, 101301

Pooley, D. \& Hut, P. 2006, ApJ, 646, L143

Rajagopal, M. \& Romani, R. W. 1996, ApJ, 461, 327

Rawls, M. L., Orosz, J. A., McClintock, J. E., Torres, M. A. P., Bailyn, C. D., \& Buxton, M. M. 2011, ApJ, 730, 25

Rutledge, R. E., Bildsten, L., Brown, E. F., Pavlov, G. G., \& Zavlin, V. E. 1999, ApJ, 514, 945

-. 2002a, ApJ, 578, 405

Rutledge, R. E., Bildsten, L., Brown, E. F., Pavlov, G. G., Zavlin, V. E., \& Ushomirsky, G. 2002b, ApJ, 580, 413

Servillat, M., Heinke, C. O., Ho, W. C. G., Grindlay, J. E., Hong, J., van den Berg, M., \& Bogdanov, S. 2012, MNRAS, 423, 1556 Servillat, M., Webb, N. A., Lewis, F., Knigge, C., van den Berg, M., Dieball, A., \& Grindlay, J. 2011, ApJ, 733, 106

Steiner, A. W., Lattimer, J. M., \& Brown, E. F. 2010, ApJ, 722, 33

一. 2012, arXiv:1205.6871

Suleimanov, V., Poutanen, J., Revnivtsev, M., \& Werner, K. 2011, ApJ, 742, 122

Tsujimoto, M. et al. 2011, A\&A, 525, A25

Verbunt, F. 2001, A\&A, 368, 137

Webb, N. A. \& Barret, D. 2007, ApJ, 671, 727

Zamfir, M., Cumming, A., \& Galloway, D. K. 2012, ApJ, 749, 69

Zampieri, L., Turolla, R., Zane, S., \& Treves, A. 1995, ApJ, 439, 849

Zavlin, V. E., Pavlov, G. G., \& Shibanov, Y. A. 1996, A\&A, 315, 141 